of which have also been isolated from CF patients from other UK hospitals. Isolates from the remaining 3 patients were unique. One additional isolate was identified as M. abscessus subsp.bolletii. No clear epidemiological connexions between patients within each cluster at our centre have been identified to date.

Conclusion These results provide further evidence that some strains of M. abscessus complex may be isolated from multiple CF patients. However, there were no clear epidemiological connexions between patients within clusters at our centre. The same strains have been isolated from patients at different UK CF centres. Further studies are required to determine the mode of acquisition of infection with these strains and whether there is a common environmental source of infection or cross infection between patients.

\section{P200 PRELIMINARY EVALUATION OF THE FUNGAL AIRWAY MICROBIOME IN ADULT CYSTIC FIBROSIS BY NEXT- GENERATION SEQUENCING, CULTURE AND STAINING TECHNIQUES}

${ }^{1} \mathrm{IC}$ Felton, ${ }^{1} \mathrm{~S}$ Benson, ${ }^{1} \mathrm{~A}$ Nicholson, ${ }^{1} \mathrm{~K}$ Al-Shafi, ${ }^{2} \mathrm{P}$ James, ${ }^{2} \mathrm{MJ}$ Cox, ${ }^{2} \mathrm{AJ}$ Walley, ${ }^{2} \mathrm{MF}$ Moffatt, ${ }^{1} \mathrm{D}$ Bilton, ${ }^{1} \mathrm{MR}$ Loebinger, ${ }^{1} \mathrm{NJ}$ Simmonds, ${ }^{2}$ WO Cookson. ${ }^{1}$ Royal Brompton and Harefield NHS Foundation Trust, London, UK; ${ }^{2}$ National Heart and Lung Institute, Imperial College London, London, UK

\subsection{6/thoraxjnl-2014-206260.329}

Introduction The prevalence and diversity of fungal airway isolates is increasing in cystic fibrosis (CF). Amidst an extending spectrum of fungal complications, lack of standardised mycology methods and poor sensitivity of culture-dependent techniques renders interpretation of isolates challenging.

Aims To evaluate the diagnostic utility of fungal cytology and microbiology stains in addition to prolonged sputum culture from adult CF patients in comparison to standard mycology techniques.

Secondly, to develop a novel, next-generation sequencing assay targeting the ITS2 region of the fungal ribosomal-RNA gene to comprehensively profile the sputum fungal microbiota.

Methods Sputum samples were investigated by a panel of three mycology techniques: prolonged fungal culture (each examined at: Day7, D14, D21, D28); Calcofluor White (CFW) stain; Grocott's Methenamine Silver (GMS) stain. A cohort of samples was also subject to broad-spectrum fungal next-generation sequencing.

Results 25 adult patients provided 45 sputum samples. Four fungal species were cultivatable: Candida species (26.6\%); Aspergillus fumigatus (4.4\%); Scedosporium apiospermum (15.5\%) and Exophiala dermatitidis (11.1\%).

Prolonged culture significantly increased overall fungal prevalence by $22 \%$ compared to standard duration (D7) $\mathrm{p}=0.008$ ). A significant increase of $11.1 \%$ in $S$. apiopspermum prevalence was observed $\mathrm{p}=0.02$ ), whilst all $E$. dermatitidis isolates required prolonged culture. The sensitivity of GMS and CFW stains $(85 \%$ and $93 \%)$ compared favourably to standard duration culture (29\%).

DNA extracted from a pilot group of these sputum samples ( $n=14 / 45)$ was subject to PCR using barcode-indexed ITS2 primers designed for Illumina-MiSeq amplicon sequencing. Fungal taxa were detected in all samples, of which seven samples (50\%) were negative after prolonged culture. Preliminary sequencing analysis of an extended sample cohort $(n=30)$ has detected 89 fungal taxa, from which only four species were cultured.
Conclusions Prolonged fungal culture is associated with a significant increase in fungal prevalence. The increased sensitivity is restricted to less common filamentous fungi associated with increasing pathogenicity: S. apiospermum and E. dermatitidis. The predictive value of stains in identifying samples positive at prolonged culture, but negative at standard duration illustrates their clinical utility.

Illumina-MiSeq ITS2-amplicon sequencing directly from sputum has identified a more diverse CF airways fungal microbiota. Preliminary analysis suggests that this is a highly sensitive tool for detecting fungi from sputum, including species which are refractory to standard and enhanced culture.

\section{P201 PNEUMOCYSTIS JIROVECII PREVALENCE IN A LARGE UK ADULT CYSTIC FIBROSIS CENTRE}

${ }^{1} \mathrm{HD}$ Green, ${ }^{1} \mathrm{R}$ Bright-Thomas, ${ }^{1} \mathrm{PJ}$ Barry, ${ }^{1} \mathrm{~A}$ Horsley, ${ }^{2} \mathrm{~K}$ Mutton, ${ }^{1} \mathrm{AM}$ Jones. ${ }^{1}$ Manchester Adult Cystic Fibrosis Centre, University Hospital of South Manchester, Manchester, UK; ${ }^{2}$ Clinical Virology Department, Central Manchester University Hospitals NHS Trust, Manchester, UK

\subsection{6/thoraxjnl-2014-206260.330}

Introduction and objectives Pneumocystis jirovecii $(P J)$ is an atypical fungus that causes pneumonia in immunocompromised patients. Its role in patients with cystic fibrosis $(\mathrm{CF})$ is unclear. Its reported prevalence in CF ranges from 1-22\% but has never been determined in the UK. Here we present preliminary crosssectional data from an ongoing study at a UK adult CF centre

Abstract P201 Table 1 Comparison of characteristics for patients with positive and negative samples

\begin{tabular}{|c|c|c|c|}
\hline \multicolumn{4}{|l|}{ Pneumocystis Jiroveccii result } \\
\hline & Negative & Positive & Sig. \\
\hline Number & 93 & 7 & NS \\
\hline \multicolumn{4}{|l|}{ Demographics } \\
\hline Male gender, n (\%) & $52(55.9)$ & $4(57.1)$ & NS \\
\hline Mean age (yr), SD & $33.8 \pm 11.1$ & $32.5 \pm 10.1$ & NS \\
\hline \multicolumn{4}{|c|}{ Spirometry and laboratory results } \\
\hline Mean $\mathrm{FEV}_{1}$ (\% predicted), SD & $56 \pm 20.1$ & $67 \pm 29.5$ & NS \\
\hline Mean BMI $\left(\mathrm{kg} / \mathrm{m}^{2}\right)$ & $22.1 \pm 3.2$ & $22.2 \pm 2.1$ & NS \\
\hline WCC $\left(x 10^{9} / L\right)$ at recruitment & $9.3 \pm 3.1$ & $9.3 \pm 2.8$ & NS \\
\hline CRP (mg/L) at recruitment & $13.0 \pm 16.8$ & $7.9 \pm 7.5$ & NS \\
\hline \multicolumn{4}{|l|}{ Chronic colonisation, n (\%) } \\
\hline Pseudomonas aeruginosa & $59(63.4)$ & $5(71.4)$ & NS \\
\hline Staphylococcus aureus & $24(25.8)$ & $1(14.3)$ & NS \\
\hline B. cepacia complex & $14(10.3)$ & $2(28.6)$ & NS \\
\hline Achromobacter xylosoxidans & $6(6.4)$ & 0 & NS \\
\hline Ralstonia species & $5(5.4)$ & 0 & NS \\
\hline NTM & $5(5.4)$ & $1(14.3)$ & NS \\
\hline Aspergillus fumigatus & $4(4.3)$ & $1(14.3)$ & NS \\
\hline Pandoraea species & $1(1.1)$ & $1(14.3)$ & NS \\
\hline MRSA & $1(1.1)$ & $1(14.3)$ & NS \\
\hline \multicolumn{4}{|l|}{ Treatment, n (\%) } \\
\hline Inhaled antibiotics & $72(77.4)$ & $6(85.7)$ & NS \\
\hline Inhaled steroids & $59(63.4)$ & $6(85.7)$ & NS \\
\hline Oral steroids (continuous) & $12(12.9)$ & $1(14.3)$ & NS \\
\hline Prophylactic macrolide & $77(83.0)$ & $5(71.4)$ & NS \\
\hline Azole therapy & $8(8.6)$ & 0 & NS \\
\hline Co-trimoxazole past 3 months & $32(34.4)$ & 0 & 0.06 \\
\hline
\end{tabular}

Key: $\mathrm{SD}=$ standard deviation, $\mathrm{WCC}=$ serum white cell count, $B$.cepacia complex $=$ Burkholderia cepacia complex, NTM $=$ Non-tuberculous mycobacteria, MRSA $=$ Methicillin resistant Staphylococcus aureus, NS $=$ not significant, $\mathrm{p}$ values $<0.1$ are shown. 